\section{Revue d'ethnoécologie}

12 | 2017

La Poule. Pratiques d'élevage et histoire culturelle

\title{
Du cobaye aux nuggets
}

L'industrialisation du poulet au XXe siècle aux États-Unis

The Industrialization of the Broiler in the United States during the 20th Century

\section{Frédéric Graber}

\section{OpenEdition}

Journals

Édition électronique

URL : http://journals.openedition.org/ethnoecologie/3329

DOI : $10.4000 /$ ethnoecologie.3329

ISSN : 2267-2419

Éditeur

Laboratoire Eco-anthropologie et Ethnobiologie

Référence électronique

Frédéric Graber, «Du cobaye aux nuggets », Revue d'ethnoécologie [En ligne], 12 | 2017, mis en ligne le 18 décembre 2017, consulté le 19 avril 2019. URL : http://journals.openedition.org/

ethnoecologie/3329; DOI : 10.4000/ethnoecologie.3329

Ce document a été généré automatiquement le 19 avril 2019

\section{(c) $(1)$}

Revue d'ethnoécologie est mis à disposition selon les termes de la licence Creative Commons Attribution - Pas d'Utilisation Commerciale - Pas de Modification 4.0 International. 


\title{
Du cobaye aux nuggets
}

\author{
L'industrialisation du poulet au $\mathrm{XX}^{\mathrm{e}}$ siècle aux États-Unis \\ The Industrialization of the Broiler in the United States during the 20th Century
}

Frédéric Graber

1 Les réacteurs, développés pendant la seconde guerre mondiale, ont progressivement remplacé les moteurs à hélices sur la plupart des avions de ligne à partir des années 1950. Ces nouvelles machines présentaient toutefois quelques inconvénients: aspirant rapidement l'air par devant pour le recracher par derrière, elles pouvaient ingérer toutes sortes de choses à proximité, en particulier des oiseaux qui sont nombreux à basse altitude, lors des opérations de décollage et d'atterrissage, et qui apprécient particulièrement les grandes étendues aéroportuaires. Des accidents spectaculaires dans les années 1960 ont amené les organismes régulateurs de l'aviation à exiger que les réacteurs passent des tests de solidité qui, à défaut de pouvoir garantir leur intégrité en cas d'ingestion d'oiseau, étaient censés limiter au maximum les incendies et explosions menaçant directement la survie de l'avion. Depuis, tout nouveau modèle est donc soumis à un test qui consiste à faire tourner le réacteur (fixé sur le tarmac) à pleine puissance et à lui lancer, grâce à un canon spécialement conçu pour cette opération, des oiseaux à une vitesse correspondant à celle d'un avion au décollage, soit $370 \mathrm{~km} / \mathrm{h}$. Le principal oiseau utilisé dans ces tests, le plus gros, est un poulet de 1,8 kg, fraîchement tué et non plumé, alors même que ce volatile n'est presque jamais impliqué dans les situations réelles d'ingestion. Si le choix s'est néanmoins porté sur cet animal peu réaliste, c'est qu'il présente des avantages décisifs : élevé dans des conditions industrielles, il est facilement disponible et bon marché, mais il compte aussi parmi les animaux les plus standardisés, ce qui est assez important pour que les tests restent comparables entre eux (Downer 2007).

2 Il est arrivé quelque chose au poulet au cours $\mathrm{du} \mathrm{xx}^{\mathrm{e}}$ siècle, au point que l'on peut sans exagérer affirmer qu'il ne s'agit plus du même animal : entre les années 1930 et les années 1990, il devient deux fois plus gros, en deux fois moins de temps, avec deux fois moins de nourriture (Boyd 2001: 638). Cette évolution est le résultat d'un processus d'industrialisation du poulet, que l'on peut suivre dans la plupart des pays occidentaux, mais qui a commencé aux États-Unis et qui s'y est développé dans des conditions tout à 
fait exceptionnelles. Cet article souhaite donc se focaliser sur le cas états-unien, en présentant dans un premier temps les grandes transformations techniques qui ont rendu possible l'industrialisation du poulet, puis, dans un second temps, le contexte spécifique dans lequel elle s'est développée aux États-Unis au cours du $\mathrm{xx}^{\mathrm{e}}$ siècle.

\section{Comment on invente un nouvel animal : le poulet}

3 Au tournant du $\mathrm{XIX}^{\mathrm{e}}$ et $\mathrm{du} \mathrm{Xx}^{\mathrm{e}}$ siècle, toute une série d'animaux ont été soumis à des exigences d'uniformité à mesure qu'émergeait la biologie expérimentale : principalement sciences de l'observation, les sciences biologiques deviennent de plus en plus expérimentales dans les dernières décennies du xix ${ }^{e}$ siècle. À partir des années 1910, on assiste en particulier à une multiplication des travaux de standardisation d'animaux de laboratoire, typiquement la drosophile dans le laboratoire de Thomas H. Morgan (et de ses élèves) ou la souris blanche de Clarence D. Little au Jackson Laboratory. La réduction au minimum des variations naturelles entre individus d'une même variété était en effet une condition essentielle pour expérimenter sur le vivant: il fallait pouvoir affirmer avec certitude que les écarts de résultats entre plusieurs expériences n'étaient pas dus à la variété naturelle des animaux, mais que ces cobayes étaient le plus identiques possible (Kohler 1994, Gaudillière 1997). Bien que dans des proportions moindres, le poulet va lui aussi connaître une certaine standardisation au $\mathrm{xx}^{\mathrm{e}}$ siècle, impulsé par une convergence d'intérêts économiques, politiques et scientifiques.

4 La poule était déjà, à la fin du xix ${ }^{\mathrm{e}}$ siècle, un animal de laboratoire, en particulier en nutrition, en bactériologie, etc. Dans les années 1900, une nouvelle science appliquée émerge, la poultry science, au croisement de nombreux savoirs qui s'intéressent à la poule ou qui la prennent comme cobaye, depuis l'économie jusqu'à l'épidémiologie en passant par les travaux universitaires sur les méthodes d'élevage (Branion \& Kent 1958). Quand l'industrie du poulet commence à émerger dans les années 1920-1930, la poule est - et de très loin - l'animal le plus étudié et le mieux connu (Boyd 2001 : 634).

5 L'investissement en recherches dans ce domaine est ancien et, dans un premier temps, massivement public. Le Morill Act de 1862, en consacrant de vastes étendues de terres fédérales à la fondation de land grant universities, a eu une influence considérable sur l'histoire américaine en promouvant des institutions de recherche et d'enseignement supérieurs orientées directement vers des préoccupations pratiques, industrielles et agricoles. Dès les années 1860, plusieurs universités fondent des laboratoires d'aviculture, mais le mouvement s'intensifie vraiment à partir de 1887 avec le Hatch Act qui permet le financement fédéral de stations expérimentales agricoles (agricultural experimental stations ) au sein des land grant universities. Le rôle de l'administration fédérale, du United States Department of Agriculture (USDA), va croissant en la matière, en particulier à partir du Smith-Lever Act de 1914, qui institue une coopération entre le USDA et les universités, les Cooperative Extension Services, qui visent à informer les agriculteurs des recherches et développements en cours, à promouvoir de nouvelles pratiques et outils et à gérer les questions et problèmes qui émergent sur le terrain. C'est surtout via ces Extension Services que l'industrialisation du poulet va progressivement être promue en lien étroit avec les grands acteurs privés du secteur. La domination de la recherche publique commence à basculer après la seconde guerre mondiale, et l'investissement de recherche devient massivement privé à partir des années 1960, correspondant aux mouvements de concentration et d'intégration verticale du secteur avicole. 
Cette poultry science impose, au cours du siècle, plusieurs grandes transformations qui ont permis l'intensification de la production, l'uniformisation des produits et la réduction massive des coûts.

7 Pour faire le poulet industriel, il a tout d'abord fallu augmenter la concentration: le poulet, que l'on élève principalement en extérieur jusqu'au début $\mathrm{du} \mathrm{xx}^{\mathrm{e}}$ siècle, vit désormais en intérieur, une concentration qui permet de mieux contrôler les bêtes et, surtout, de réduire au minimum les fonctions corporelles, l'énergie dépensée à s'égayer en plein air étant perdue pour la croissance optimisée de l'animal. Malheureusement les poulets ne sont pas faits pour rester à peu près immobiles en intérieur pendant des semaines: confinés, ils ont tendance à développer une forme de rachitisme, la leg weakness. Dans les années 1920, plusieurs travaux ont montré que l'on peut éviter ces affections en introduisant de l'huile de foie de morue dans l'alimentation des poulets et en les soumettant à des lampes UV : ces travaux, qui s'inscrivent dans une histoire longue de la découverte et des recherches sur le fonctionnement de la vitamine $\mathrm{D}$, ont permis de dépasser une des principales limitations à l'élevage intensif et d'envisager, surtout aprèsguerre, des formes de plus en plus extrêmes de concentration (Boyd $2001: 638$ ).

Pour faire le poulet industriel, il a également fallu séparer la production des poulets de celles des œufs et de leur incubation. Jusqu'au début du $\mathrm{xx}^{\mathrm{e}}$ siècle, le poulet est un dérivé de la filière œuf (déjà en cours d'industrialisation à la fin du xIX siècle) : dans la sélection des poules pondeuses, les coqs n'étant d'aucune utilité, on commercialise les plus jeunes et les plus tendres comme poulets à rôtir. À mesure que ce poulet émerge comme produit autonome, on sélectionne des types de poules particuliers pour leur chair et non plus pour leurs œufs : le poulet (broiler) est né, avec comme conséquence secondaire que, dans l'industrie de l'œuf, les mâles deviennent non seulement inutiles (non pondeurs) mais invendables par rapport aux nouveaux venus et par conséquent détruits en bas âge. La sélection des poulets pour leurs traits les plus intéressants (grosses cuisses et poitrines) s'intensifie au cours du $\mathrm{xx}^{\mathrm{e}}$ siècle et est pour beaucoup dans le doublement du poids des poulets moyens au cours du siècle. Les compagnies spécialisées dans l'élevage et l'incubation de ces nouvelles variétés vont aussi favoriser les hybrides, non seulement pour leurs qualités propres mais aussi parce qu'ils permettent d'imposer une forme de propriété sur le vivant, une « fermeture biologique » : les hybrides se reproduisent mal ou perdent leurs qualités à la reproduction, les fermiers sont donc obligés de racheter des poussins et ne peuvent monter leur propre filière d'incubation (Boyd 2001: 658, Derry 2012).

9 Pour faire le poulet industriel, il a encore fallu organiser une gestion sanitaire: la concentration des poulets favorise, en effet, la transmission de maladies. Un fort investissement de recherche a fait émerger des outils pharmaceutiques contre les maladies les plus courantes chez le poulet et a permis de maintenir des concentrations élevées sans faire périr l'ensemble du cheptel. En opérant quotidiennement des tours de surveillance pour éliminer au plus vite les éléments malades, les éleveurs pouvaient espérer limiter au maximum les contagions et les pertes. Les pratiques d'élevage industriel ont consisté à intégrer statistiquement la maladie dans le processus de production: on ne peut descendre en dessous d'une certaine proportion de malades, mais, en adoptant des pratiques vigilantes, on peut se rapprocher le plus possible de ce seuil incompressible. Dès les années 1940, la poultry science a cherché à utiliser des antibiotiques de manière préventive pour faire baisser ce seuil: ces recherches ont permis d'établir qu'indépendamment de ces effets sanitaires, l'addition d'antibiotiques à 
l'alimentation du poulet permet d'obtenir un gain de poids d'environ $10 \%$ à alimentation égale. En 1951, la Food and Drug Administration autorise l'utilisation de plusieurs antibiotiques comme additifs alimentaires agricoles dont l'emploi va se généraliser aux États-Unis, puisqu'ils constituent des accélérateurs de croissance très bon marché. Malgré des conséquences sur l'émergence de souches microbiennes résistantes, cette consommation d'antibiotique est devenue incontournable pour l'économie du poulet ${ }^{1}$ (Boyd 2001 : 647).

10 Pour faire le poulet industriel, enfin, il a fallu changer assez radicalement son alimentation. Le poulet était déjà l'un des principaux cobayes dans les recherches nutritionnelles de la fin $\mathrm{du} \mathrm{XIX}^{\mathrm{e}}$ siècle, en particulier pour étudier les fonctions physiologiques de certains nutriments. Au cours des années 1930 et 1940, la poultry science a permis un saut dans l'optimisation du rapport entre la croissance des poulets et le volume (le coût) de leur alimentation: en jouant sur les rapports énergie/fibres et protéine/fibres, les chercheurs ont établi qu'une combinaison entre maïs et soja permet d'obtenir une croissance rapide à moindre coût. Ces deux plantes se sont alors imposées après la seconde guerre mondiale comme les incontournables de l'alimentation animale, transformant l'agriculture aux États-Unis et bientôt à l'échelle mondiale: le maïs en particulier est planté à peu près partout, même dans des contextes très défavorables (Boyd 2001 : 645-646).

\section{D'un plat du dimanche à la viande de tous les jours}

11 Le poulet est aujourd'hui la première viande consommée aux États-Unis: chaque Américain en consomme en moyenne $45 \mathrm{~kg}$ par an; en 1934, c'était tout juste $300 \mathrm{~g}^{2}$. Au début du siècle, le poulet n'était pas considéré comme une viande, à l'égal du porc ou du bœuf : c'était un plat exceptionnel, cher, que l'on mangeait dans les grandes occasions. En 1928, avant la crise, le parti républicain pouvait choisir comme slogan pour la campagne présidentielle "A chicken for every pot » : cela n'avait encore rien d'évident qu'il $\mathrm{y}$ ait un poulet dans toutes les marmites ${ }^{3}$. Comment ce poulet du dimanche est-il devenu une viande, et même la viande par excellence?

Dans la seconde moitié du XIX ${ }^{\mathrm{e}}$ siècle, le commerce de la viande de bœuf et de porc s'est à la fois considérablement intensifié et centralisé aux États-Unis: de très grosses compagnies prennent progressivement le contrôle d'une bonne partie de la chaîne de production, depuis le transport des animaux vivants, l'abattage, la découpe, le conditionnement et le transport vers les consommateurs. Ces meat-packers sont concentrés dans quelques grands centres urbains - Chicago au premier chef, qui capte l'essentiel de la production de l'Ouest américain - d'où ils expédient la viande en wagons réfrigérés pour alimenter l'ensemble des villes de la côte Est (Cronon 1991 : 207-259). Dans les années 1880, ces meat-packers se sont positionnés sur le marché de l'œuf et ont essayé d'étendre leur modèle avec le poulet. Mais l'atout qu'offrent les wagons réfrigérés pour transporter des poulets préparés vers les grands centres urbains de la côte Est s'est avéré insuffisant, face à la saisonnalité du produit, aux variations importantes de qualité, à une très forte décentralisation de la production et à une nette préférence, au tournant $\mathrm{du} \mathrm{XIX}^{\mathrm{e}}$ et du Xx $\mathrm{x}^{\mathrm{e}}$ siècle, pour l'achat de poulets vivants. Le poulet a donc suivi une autre voie. 
13 L'industrialisation du poulet commence dans les années 1920, principalement dans la péninsule du Delmarva (Delaware, Maryland et Virginia). La proximité des grands centres urbains (Philadelphie et New York au nord, Baltimore et Washington à l'ouest) y a favorisé l'émergence d'élevages intensifs de poulets, qui pouvaient être acheminés vivants par camion ou chemin de fer. Ce type de transport s'avère difficile sur de longues distances: les sites de production ne pouvaient donc se trouver très éloignés des consommateurs (Striffler 2005 : 34). Dans les années 1920, plusieurs fermiers du Delmarva ont tenté avec succès la commercialisation de leurs poulets à New York et ont ensuite augmenté spectaculairement leurs capacités de production, passant de quelques centaines à quelques dizaines de milliers : le succès des premiers a entraîné les suivants et, en l'espace de quelques années, un territoire entier s'est spécialisé dans le poulet, auquel il a donné une dimension véritablement industrielle. Si les fermiers du Delmarva se sont engagés dans une production massive de poulets, c'est avant tout parce New York s'est mis à les consommer dans les mêmes proportions. New York est, au début du siècle, la plus grosse consommatrice de poulet, non seulement du fait de sa taille (c'est de très loin la plus grosse ville américaine), mais surtout grâce à l'importance de sa population juive, en forte croissance au tournant des $\mathrm{XIX}^{\mathrm{e}}$ et $\mathrm{Xx}^{\mathrm{e}}$ siècles ${ }^{4}$. Selon une enquête de l'administration fédérale en 1926, $80 \%$ des poulets vendus vivants à New York étaient achetés par des juifs, qui privilégiaient les poulets par rapport aux autres viandes précisément parce qu'ils étaient importés vivants et pouvaient donc être tués conformément aux prescriptions d'un abattage rituel ${ }^{5}$ (Horowitz 2004 : 217).

$\mathrm{Au}$ cours des années 1930, cette consommation de poulets vivants va être progressivement détrônée par le New-York dressed, qui séduit un nombre de plus en plus important de consommateurs qui ne veulent plus gérer l'abattage ou sont moins regardants sur les rituels : il s'agit d'un poulet tué, plumé, mais non éviscéré, transporté par wagons (ou camions) réfrigérés. Les usines de processing (abattage et conditionnement) se multiplient dans le Delmarva, particulièrement à partir de la fin des années 1930, reflétant cette évolution (Williams 1998 : 31-32.)

La seconde guerre mondiale a profondément perturbé cette industrie émergente. En 1942, la War Food Administration réquisitionne pour l'alimentation des troupes toute la production de la péninsule du Delmarva, qui s'était spécialisée sur ce créneau et occupait de très loin la première place en tant que producteur. L'essentiel de la production de poulet disparaît donc du marché, mais les consommateurs eux ne disparaissent pas, ils vont même se faire de plus en plus nombreux: le poulet est, en effet, une des rares viandes à ne pas être soumise au rationnement pendant la guerre, ce qui va contribuer à la lancer comme viande de consommation courante. La réquisition des poulets du Delmarva va être une aubaine pour plusieurs États qui vont développer une industrie de remplacement pendant la guerre. Ces nouveaux producteurs (et conditionneurs) émergent principalement dans les États du Sud, dans une configuration économique et sociale tout à fait particulière : les petits agriculteurs ont été durement frappés par le Dust Bowl et par la crise, ils ne peuvent plus tenir la concurrence contre l'émergence d'une agriculture industrielle qui exige des capitaux importants et ils fournissent aussi une main-d'œuvre précarisée disponible à bon marché pour des opérations de processing (Striffler 2005 : 43-45 ; Boyd \& Watts 1997 : 153). Ce déplacement géographique a eu un impact à long terme : aujourd'hui encore, l'essentiel de la production de poulet et toutes les opérations annexes de conditionnement ont lieu dans les États du Sud, principalement en (par ordre décroissant d'importance) Georgie, Alabama, Caroline du Nord, Arkansas, 
Mississippi, Texas, etc. (USDA 2016 : 6-7). Depuis la seconde guerre mondiale, le poulet est installé dans des régions défavorisées comptant de nombreux travailleurs pauvres (Striffler $2005: 36$ ).

16 Après la guerre, la filière poulet, travaillant main dans la main avec l'administration fédérale, le USDA, va investir massivement pour faire du poulet un plat de tous les jours : on cherche alors surtout à faire baisser les prix en sélectionnant les meilleurs types et en améliorant leur état sanitaire; on cherche aussi à obtenir une qualité uniforme pour standardiser les attentes des consommateurs (principalement en mettant un terme à la saisonnalité du produit et en faisant converger les différents types de poulets); on cherche enfin à promouvoir le poulet non plus comme un produit de luxe, mais comme une consommation commune.

17 Ce programme passe en particulier par l'organisation de concours régionaux ou nationaux, dont le plus célèbre est le Chicken of Tomorrow Contest, initié et financé par la grande chaîne de magasins d'alimentation A\&P avec la collaboration du USDA. Organisé d'abord au niveau régional en 1946 et 1947, il permet de dégager quarante finalistes pour le concours national organisé en 1948 : toutes les grandes hatcheries, les compagnies d'élevage et d'incubation, fournissent les œufs qui sont anonymisés, incubés, puis élevés dans des conditions uniformes à la station agricole expérimentale du Delaware, la nourriture standardisée étant mise à disposition par l'American Feed Manufacturers Association $^{6}$. Ce genre de concours a eu un impact considérable pour l'uniformisation des types de poulet et des modes d'élevage. Dans les dix années qui suivent le Chicken of Tomorrow Contest de 1948, les poulets du vainqueur, Vantress Hatcheries de Californie, réunissant le meilleur rapport croissance/nourriture, vont s'imposer comme le standard pour l'ensemble de la filière (Horovitz 2004 : 215).

18 Cette convergence des pratiques de production et des races utilisées passe aussi par la création de marchés spécialisés, promus par l'administration, typiquement la Eastern Shore Poultry Exchange (1952-1969), où les acheteurs de poulets, les conditionneurs, imposent progressivement leurs attentes. Le succès de Vantress Birds, qui devient le fournisseur de poussins le plus demandé au cours des années 1950, est typique de ce point de vue : les éleveurs sont d'abord plutôt réticents à acheter ces nouveaux oiseaux, plus chers, mais les acheteurs imposent ce type de poulet dans les transactions des marchés spécialisés, après le succès du Chicken of Tomorrow Contest de 1948.

19 Ces marchés spécialisés, en favorisant des concentrations du côté des compagnies d'élevage et d'incubation, vont aussi faire émerger des acteurs qui prétendent gérer l'ensemble des opérations de la filière : les intégrateurs (integrators) dont les plus célèbres, comme Perdue et Tyson, sont encore aujourd'hui des acteurs centraux du marché du poulet. Ceux-ci vont généraliser le principe du contracting farming, dans lequel les éleveurs de poulets reçoivent de la compagnie poussins et nourriture, lui fournissent en retour les poulets, les pratiques d'élevage étant soumises au contrôle d'inspecteurs-fournisseurs, les servicemen. Ce système permet aux compagnies d'externaliser l'essentiel des risques (principalement sanitaires) sur les éleveurs, précarisés et dépendants, tout en contrôlant toute la chaîne de production. L'importance que vont prendre ces intégrateurs dans les années 1960 se traduit par la disparition des marchés spécialisés, devenus finalement inutiles, puisque leurs contractants représentent l'essentiel de la production ${ }^{7}$ (Horowitz $2004: 221)$. oiseaux, nouvelles nourritures, etc.) exigent des investissements importants en capital, 
que les éleveurs majoritairement modestes ne peuvent obtenir des banques qu'au prix de très lourds intérêts : ils vont donc être séduits par un système qui leur fournit tous les éléments dont ils ont besoin (poussins, nourriture, expertises et conseils), sans percevoir au premier abord les dépendances fortes et irréversibles dans lesquelles ils s'engagent, en particulier via des crédits qu'ils peineront à rembourser au regard des faibles profits qu'ils peuvent espérer - les prix des intrants comme des produits étant de facto contrôlés par l'intégrateur (Striffler 2005 : 49-50). Jusqu'aux années 1950, la filière poulet comptait des acteurs très nombreux : à la fin des années 1960, il ne reste plus que quelques très gros intégrateurs (Boyd \& Watts 1997 : 145).

21 Au cours des années 1960-1970, le poulet devient un objet publicitaire, à mesure que les grands groupes qui dominent ce marché entendent faire évoluer les consommations et que les profits considérables qu'ils dégagent leur permettent d'envisager des campagnes publicitaires coûteuses (Striffler 2005 : 24). Au début des années 1960, le poulet n'est toujours pas véritablement considéré comme une viande, principalement à cause de la monotonie des produits comparativement aux viandes de porc et de bœuf (Horowitz 2004 : 224). À mesure que le poulet est consommé plus fréquemment, des phénomènes de lassitude émergent chez les consommateurs, que l'industrie du poulet va chercher à tromper. C'est en particulier la stratégie adoptée par Tyson, qui consiste à proposer le poulet sous des formes et des préparations multipliées : morceaux choisis, précuits ou non, plats préparés, etc. S'amorce ici une des principales évolutions dans les formes de consommation du poulet: au début des années 1960 le poulet se vendait encore principalement entier aux États-Unis (à $83 \%$ ), un conditionnement qui ne représentait plus que $14 \%$ en 1995. Au début du XxI ${ }^{\mathrm{e}}$ siècle, l'essentiel du poulet consommé aux ÉtatsUnis l'est sous des formes fragmentaires et transformées. Cette multiplication des produits n'a pas seulement permis de dépasser la lassitude des consommateurs, elle a surtout rendu le poulet plus profitable, en créant de la valeur ajoutée sur un produit aux marges très serrées (Striffler $2005:$ 21).

Dans ces mêmes années, la publicité promeut le poulet comme une viande saine, maigre, dans un contexte de plus en plus attentif à la nutrition - ce qui est d'autant plus ironique que le poulet est de plus en plus massivement commercialisé par les grandes chaînes de fast-food, souvent sous forme de fritures. Parallèlement, les publicitaires appliquent la stratégie du branding au poulet en cherchant à associer un nom et une marque à une certaine idée de qualité. C'est le cas en particulier de Perdue, dont les campagnes des années 1970 comptent parmi les plus grands succès de l'histoire de la publicité, lui permettant de passer en quelques années de $1 \%$ à $25 \%$ du marché. La stratégie de Perdue a consisté à la fois à se mettre en scène lui-même (comme inspecteur et garant de la qualité de ses poulets) et à mettre en avant un signe facilement reconnaissable (la couleur jaune) de la qualité prétendument supérieure de ses animaux (Striffler 2005 : 24-25).

Une publicité très célèbre pour Kentucky Fried Chicken [KFC] en 1967 présente sept boîtes de wings frits avec comme sous-titre: We fix Sunday dinner on Monday, Tuesday, Wednesday, Thursday, Friday, Saturday and Sunday. La multiplication des produits et des marques va permettre non seulement de faire du poulet une viande de tous les jours, une viande comme les autres, mais même d'en faire la viande la plus consommée aux États-Unis, ce seuil étant atteint dans les années 1980. L'industrie du fast-food y a été beaucoup, KFC tout d'abord, rejoint par McDonald's dans les années 1980, l'introduction des McNuggets (1983) ayant propulsé ce dernier comme l'un des premiers utilisateurs mondiaux de poulet. Les nuggets représentent un tournant important dans l'histoire du poulet: rapidement 
devenu l'article préféré des enfants, il a permis un développement du fast-food spécifiquement à destination de ces petits consommateurs, mais il orienté l'ensemble de la filière puisque les nuggets sont devenus la forme standard de consommation du poulet aux États-Unis au tournant du XxI e siècle (Striffler 2005 : 17-18, 27).

Cette généralisation des produits dérivés a eu des conséquences sur le processing. La maind'œuvre était déjà très importante dans l'industrie du poulet depuis l'après-guerre, surtout depuis les années 1950 et la généralisation de l'éviscération. Mais, malgré des avancées importantes en terme de mécanisation, l'abattage et surtout la préparation des produits continuent à exiger une main-d'œuvre considérable : dans les années 1960-1970, ce sont les découpes de morceaux et les préparations de plats préparés, depuis les années 1980, ce sont les produits dérivés, en particulier les nuggets, qui réclament un désossage manuel important. L'industrie du poulet est donc restée un très gros employeur, mais principalement en employant des pauvres. Les salaires y sont parmi les plus bas de l'industrie états-unienne ; les syndicats y sont inexistants ou très faibles, les grands groupes ayant mené des actions anti-syndicats illégales, mais souvent avec succès ${ }^{8}$ ; les taux d'accident y ont toujours été particulièrement élevés; le turnover y est spectaculaire (jusqu'à 100 \% par an), les employés ne tenant jamais très longtemps face à la fatigue, aux accidents et aux maladies résultant des opérations répétitives et dangereuses (Striffler 2005 : 75-76 et 115). L'industrie du poulet se caractérise surtout par ce qu'elle emploie toujours dans le processing les populations les plus fragiles du moment : les blancs pauvres jusqu'aux années 1950, puis principalement des noirs pauvres (1960-1970), enfin, depuis les années 1980, des latino-américains pauvres qui représentent aujourd'hui près des trois quarts des employés des usines (Horowitz 2004: 230 ; Striffler 2005 : 94 et 98-99). Plusieurs grandes compagnies ont été impliquées dans des trafics d'immigrants (Tyson en 2001), allant jusqu'à élaborer de véritables filières pour leur faire traverser la frontière mexicaine, leur procurer de faux papiers, afin d'obtenir une maind'œuvre meilleur marché, prête à accepter des conditions de travail plus difficiles. Les sans-papiers sont devenus essentiels dans cette industrie: eux seuls permettent de maintenir le prix dérisoire du poulet qui arrive sur les tables américaines (Striffler 2005 : 112).

\section{BIBLIOGRAPHIE}

Boyd W. \& Watts M. 1997 - Agro-industrial Just-in-Time. The Chicken Industry and Postwar American Capitalism. In : Goodman D. \& Watts M. (Ed.), Globalising Food. Agrarian Questions and Global Restructuring. New York, Routledge : 139-165.

Boyd W. 2001 - Making Meat. Science, Technology, and American Poultry Production. Technology and Culture $42: 631-664$.

Branion H.D. \& Kent O.B. 1958 - A Brief History of the Poultry Science Association and its Journal, 1908-1958. Ithaca, Cornell University, 41 p. 
Cronon W. 1991 - Nature's Metropolis. Chicago and the Great West. New York \& Londres, Norton, $592 \mathrm{p}$.

Derry M.E. 2012 - Art and Science in Breeding. Creating Better Chickens. Toronto, University of Toronto Press, 228 p.

Downer J. 2007 - When the Chick Hits the Fan: Representativeness and Reproducibility in Technological Tests. Social Studies of Science 37 (1) : 7-26.

Gaudillière J.-P. 1997 - Des souris « inbred » et des hommes. Pour la pathologie et la génétique, des millions de souris presque identiques. La Recherche 300 : 94-98.

Horowitz R. 2006 - Putting Meat on the American Table: Taste, Technology, Transformation. Baltimore, The John Hopkins University Press, 192 p.

Horowitz R. 2004 - Making the Chicken of Tomorrow: Reworking Poultry as Commodities and Creatures, 1945-90. In : Schrepfer S.R. \& Scranton P. (Ed.), Industrializing Organisms: Introducing Evolutionary History. New York, Routledge : 215-235.

Kohler R.E. 1994 - Lords of the Fly. Drosophila Genetics and the Experimental Life. Chicago, The University of Chicago Press, $344 \mathrm{p}$.

Ritterband P. - Counting the Jews of New York, 1900-1991: An Essay in Substance and Method. Jewish Population Studies (Papers in Jewish Demography) 29 : 199-228.

Striffler S. 2005 - Chicken. The Dangerous Transformation of America's Favorite Food. New Haven, Yale University Press, 208 p.

USDA 2016 - United States Department of Agriculture. Poultry - Production and Value. 2015 Summary.

Williams W.H. 1998 - Delmarva's Chicken Industry: 75 Years of Progress. Georgetown (Delaware), Delmarva Poultry Industry Inc., $128 \mathrm{p}$.

\section{NOTES}

1. En 2005, après une lutte de cinq ans avec la multinationale Bayer, la Food and Drug Administration a réussi pour la première fois à interdire l'emploi d'un antibiotique comme additif pour l'alimentation des poulets, parce que cet usage menace de rendre cet antibiotique inefficace pour l'homme face à des souches microbiennes désormais résistantes. « Ending Battle With FDA, Bayer Withdraws Poultry Antibiotic», Washington Post, 9 septembre 2005, http:// www.washingtonpost.com/wp-dyn/content/article/2005/09/08/AR2005090801918.html consulté le 27 juin 2016.

2. 2,3 kg en 1945, 10,7 kg en 1960, 21,2 kg en 1980 et 31,7 en 1994 (Boyd \& Watts, $1997: 140$ ).

3. «A Chicken in Every Pot ", New York Times, 30 octobre 1928. National Archives. Herbert Hoover Papers: Clippings File, 1920-1964. https://research.archives.gov/id/187095, consulté le 17 juin 2016.

4. Un recensement mené en 1878, avant la vague d'immigration de juifs d'Europe de l'Est, par deux institutions juives new-yorkaises, portait à 60000 le nombre de juifs vivants à New York et à 13000 à Brooklyn. Tous les chiffres ultérieurs sont des évaluations. En 1916, la population juive de New York est évaluée entre 1500000 et 1700000 (Ritterband, $1997: 201$ et 207).

5. Voir par exemple l'image de la photographe américaine Berenice Abbott d'un chicken market en 1937 : https://www.brooklynmuseum.org/opencollection/objects/121232/

Chicken_Market_55_Hester_Street_Manhattan 
6. L'événement a été l'objet d'une communication exceptionnelle, en particulier par la réalisation de films documentaires : voir The Chicken of Tomorrow Contest, Audio Productions, 1948, Preminger Archives : https://archive.org/details/Chickeno1948 consulté le 19 juin 2016.

7. Ce phénomène d'intégration correspond aussi à un moment de généralisation du processing. La commercialisation du poulet vivant devient marginale et le produit standard est désormais un poulet prêt à l'emploi, non seulement tué et plumé (comme l'était le New-York dressed) mais aussi éviscéré. Les opérations d'éviscération entraînent une augmentation considérable de maind'œuvre qui, de blanche, devient majoritairement noire au cours des années 1950, tout en restant essentiellement féminine.

8. Aujourd'hui encore Tyson Food accueille ses employés avec le panneau suivant apposé dans ses usines : « Les démocraties dépendent de la participation politique de leurs citoyens, mais pas sur le lieu de travail. » (Striffler $2005: 112)$.

\title{
RÉSUMÉS
}

Il est arrivé quelque chose au poulet au cours $\mathrm{du} \mathrm{XX}^{\mathrm{e}}$ siècle, au point qu'il ne s'agit plus du même animal : le poulet devient deux fois plus gros, en deux fois moins de temps, avec deux fois moins de nourriture à la fin du siècle qu'au début. Cette évolution est le résultat d'un processus d'industrialisation du poulet, que l'on peut suivre dans la plupart des pays occidentaux, mais qui a commencé aux États-Unis et qui s'y est développé dans des conditions tout à fait exceptionnelles. Cet article se focalise donc sur le cas états-unien, en présentant dans un premier temps les grandes transformations techniques qui ont rendu possible l'industrialisation du poulet, puis dans un second temps le contexte spécifique dans lequel elle s'est développée aux États-Unis au cours du $\mathrm{xx}^{\mathrm{e}}$ siècle.

Something happened to chickens during the 20th century, something so massive that they clearly became another kind of animal: broilers today get twice as big in half the time and with half the feeding they needed at the start of the century. This transformation of the broiler is the result of a process of industrialization that happened in most Western countries, but started in the United States and expanded there like nowhere else. This paper presents the major technical innovations that made the industrialization of the broiler possible in the first place and on the specific context of its expansion in the United States during the 20th century.

\section{INDEX}

Keywords : industrialization, broiler, chicken, United States, meat, nuggets

Mots-clés : industrialisation, poulet, États-Unis, viande, nuggets

\author{
AUTEUR \\ FRÉDÉRIC GRABER \\ Centre de recherches historiques, CNRS-EHESS, Paris \\ graber@ehess.fr
}

